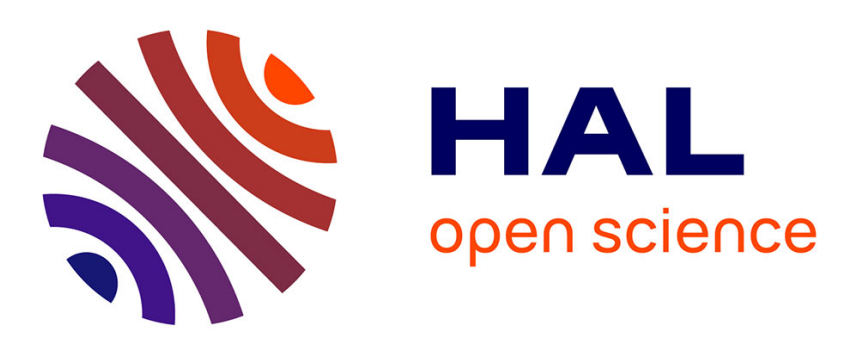

\title{
Stabilization and control Lyapunov functions for language constrained discrete-time switched linear systems
}

\author{
Mirko Fiacchini, Marc Jungers, Antoine Girard
}

\section{To cite this version:}

Mirko Fiacchini, Marc Jungers, Antoine Girard. Stabilization and control Lyapunov functions for language constrained discrete-time switched linear systems. Automatica, 2018, 93, pp.64-74. 10.1016/j.automatica.2018.03.039 . hal-01677493

\section{HAL Id: hal-01677493 https://hal.science/hal-01677493}

Submitted on 8 Jan 2018

HAL is a multi-disciplinary open access archive for the deposit and dissemination of scientific research documents, whether they are published or not. The documents may come from teaching and research institutions in France or abroad, or from public or private research centers.
L'archive ouverte pluridisciplinaire HAL, est destinée au dépôt et à la diffusion de documents scientifiques de niveau recherche, publiés ou non, émanant des établissements d'enseignement et de recherche français ou étrangers, des laboratoires publics ou privés. 


\title{
Stabilization and control Lyapunov functions for language constrained discrete-time switched linear systems
}

\author{
Mirko Fiacchini $^{\mathrm{a}}$, Marc Jungers ${ }^{\mathrm{b}}$, Antoine Girard ${ }^{\mathrm{c}}$ \\ ${ }^{a}$ Univ. Grenoble Alpes, CNRS, Grenoble INP, GIPSA-lab, 38000 Grenoble, France \\ (email: mirko.fiacchini@gipsa-lab.fr) \\ ${ }^{\mathrm{b}}$ Université de Lorraine, CRAN, UMR 7039, 2 avenue de la forêt de Haye, Vandouvre-lès-Nancy Cedex, 54516, France and CNRS, \\ CRAN, UMR 7039, France (email: marc.jungers@univ-lorraine.fr) \\ ${ }^{\mathrm{c}}$ Laboratoire des signaux et systèmes (L2S), CNRS, CentraleSupélec, Université Paris-Sud, Université Paris-Saclay, 3, rue Joliot-Curie, \\ 91192 Gif-sur-Yvette, cedex, France (email: Antoine.Girard@l2s.centralesupelec.fr)
}

\begin{abstract}
In this paper, the stabilizability of discrete-time switched linear systems subject to constraints on the switching law is considered. The admissible switching sequences are given by the language generated by a nondeterministic finite state automaton. Constructive necessary and sufficient conditions for recurrent stabilizability are given and the exact relations with the existence of control Lyapunov functions and with general stabilizability are provided. The dependence of stabilizability on the automaton initial state is also proved.
\end{abstract}

Key words: Switched linear systems; set-theory; stabilizability; automata.

\section{Introduction}

Switched systems are systems whose behavior evolves among a finite class of different dynamics, [15]. Every behavior is characterized by the active mode that is selected by a function of time, the switching law. The interest in switched systems increased in the last decades due to their capability of modelling complex systems, such as embedded and networked systems. On the other hand, the analysis and control design for switched system might be rather involved, also for linear switched systems, [15,22]. Concerning the problem of stabilizability of switched system, it is known that convex Lyapunov functions lead to conservative results, and nonconvex ones must be considered, see [6]. Nonconvex Lyapunov functions induced by the union of ellipsoids are employed in $[7,11,26,12,8]$, while more general homogeneous functions have been considered in [9].

In this paper, whose preliminary version is [10], we are considering the problem of stabilizability of switched linear systems subject to constraints on the switching law. In many practical cases, indeed, the mode sequence might be required to satisfy some conditions. Consider for instance the prob-

\footnotetext{
* Corresponding author M. Fiacchini. Tel: 33 (0)4 768262 25, fax : 33 (0)4 768263 88, email: mirko.fiacchini@gipsa-lab.fr.
}

lems of safety specifications, the tasks scheduling, the interaction between control and software implementation and the constraints on dwell-time switching. Several kinds of these constraints may be modeled by a nondeterministic finite automaton, see [3], by imposing that the switching law belongs to the language generated by such an automaton. The idea of employing regular languages and automata to impose constraints on the switching law has been recently applied to the problem of stability analysis for switched linear systems. The problem of stability of constrained switched linear system is addressed in $[25,20,24]$ using automata properties while converse Lyapunov theorems, based on the joint spectral radius approach, are provided in [18]. Graph Lyapunov functions and spectral radius are employed in [1] and in [14] directed graphs are used to determine the switching sequences under which the system is stable. LyapunovMetzler conditions are considered in [13] and invariance in [2] for constrained switched systems.

The problem of determining stabilizing feedback control policies satisfying language constraints, dealt with in this paper, has not been treated, in the authors knowledge. For this purpose, we consider nonconvex star-shaped sets, see [19], and their gauge functions as Lyapunov candidates, as in [9], to provide an algorithm leading to constructive conditions for stabilizability. The concepts of recurrent exponential stabilizability and recurrent control Lyapunov func- 
tions are introduced. The finite termination of the algorithm is proved to be necessary and sufficient, not only for the existence of a recurrent Lyapunov function, as proved in [10], but also of general Lyapunov functions and for recurrent stabilizability. Moreover we prove that recurrent stabilizability is only sufficient for stabilizability. Finally, the dependence of stabilizability on the automaton initial state is analyzed.

Notations Denote with $\mathbb{R}^{+}$the set on nonnegative real numbers. Given $n \in \mathbb{N}$, define $\mathbb{N}_{n}=\{j \in \mathbb{N}: 1 \leq j \leq n\}$. Given $\Omega \subseteq \mathbb{R}^{n}$ define the interior of $\Omega$ as $\operatorname{int}(\Omega)$, its closure as $\operatorname{cl}(\Omega)$ and its boundary as $\partial \Omega$. The Euclidean norm in $\mathbb{R}^{n}$ is $\|x\|$ and the unit ball is denoted $\mathscr{B}^{n}$. The $i$-th element of a finite set of matrices is denoted as $A_{i}$. Given a finite set $I$ and $N, M \in \mathbb{N}$ with $0<N \leq M$, all the possible sequences of length $N$ of elements of $I$ is $I^{N}=\prod_{j=1}^{N} I$; define also $I^{[N, M]}=\bigcup_{k=N}^{M} I^{k}$ and $|\sigma|=N$ if $\sigma \in I^{N}$. The $i$-th element of a sequence $\sigma$ is $\sigma_{i}$. Given $y \in Y$ and $z \in Z$, the vector $\left[y^{T}, z^{T}\right]^{T} \in Y \times Z$ is also denoted $(y, z)$. Given a matrix $A \in$ $\mathbb{R}^{n \times m}$ we use $A^{-1}$ to denote, with slight abuse of notation, both its inverse matrix, if $A$ is invertible, and the preimage of a set $\Omega \subseteq \mathbb{R}^{m}$ through $A$, that is $A^{-1} \Omega=\left\{x \in \mathbb{R}^{n}: A x \in \Omega\right\}$, well-defined even for singular $A$. The rotation matrix in $\mathbb{R}^{2}$ is $R(\theta)=\left[\begin{array}{cc}\cos (\theta) & -\sin (\theta) \\ \sin (\theta) & \cos (\theta)\end{array}\right]$ for $\theta \in \mathbb{R}$.

\section{Problem Formulation}

\subsection{Language constrained switched systems}

Consider the discrete-time switched linear system

$$
x_{k+1}=A_{\sigma_{k}} x_{k},
$$

with $x_{k} \in \mathbb{R}^{n}$ the state at time $k \in \mathbb{N}$; the set of $q$ switching modes is $\mathscr{I}=\mathbb{N}_{q} ; \sigma: \mathbb{N} \rightarrow \mathscr{I}$ the switching law and

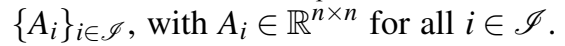

Assumption 1 The matrices $A_{i}$, with $i \in \mathscr{I}$, are nonsingular.

Assumption 1, although not necessary, is supposed to hold on the most part of the paper to maintain the results presentation simple. The proofs for the more general case of singular matrices requires the modification of some definitions, in particular concerning the Lyapunov functions, but are substantially analogous to those presented in the paper. Some considerations regarding the case of singular matrices are provided in Section 3.3.

We impose the constraint that $\sigma$ has to belong to the language specified by a nondeterministic finite automaton.

Definition 1 A nondeterministic finite automaton is a tuple $\mathscr{A}=\left(\mathscr{S}, \mathscr{I}, \delta, \mathscr{S}_{0}\right)$ where $\mathscr{S}$ is a finite set of states, $\mathscr{I}=$
$\mathbb{N}_{q}$ is a finite alphabet, $\delta: \mathscr{S} \times \mathscr{I} \rightarrow 2^{\mathscr{S}}$ is a set-valued transition map, and $\mathscr{S}_{0} \subseteq \mathscr{S}$ is a subset of initial states.

A state $s \in \mathscr{S}$ is non-blocking if there is $i \in \mathscr{I}$ such that $\delta(s, i) \neq \emptyset$, it is blocking otherwise. A switching law $\sigma: \mathbb{N} \rightarrow$ $\mathscr{I}$ belongs to the language of $\mathscr{A}$, denoted $L(\mathscr{A})$, if there is a sequence $s^{\sigma}: \mathbb{N} \rightarrow \mathscr{S}$, referred to as automaton trajectory generating $\sigma$, such that $s_{0}^{\sigma} \in \mathscr{S}_{0}$ and $s_{k+1}^{\sigma} \in \delta\left(s_{k}^{\sigma}, \sigma_{k}\right)$ for all $k \in \mathbb{N}$. A state $b \in \mathscr{S}$ is reachable from $a \in \mathscr{S}$ if there exists an automaton trajectory $s^{\sigma}$ and $N, M \in \mathbb{N}$, with $N \leq M$, such that $s_{N}=a$ and $s_{M}=b$. Given $\Omega \subseteq \mathbb{R}^{n}$ and $s \in \mathscr{S}$, we denote the set $\Omega \times\{s\}$ as $\Omega \times s$ to simplify the notation.

In the paper, we make the following assumptions on $\mathscr{A}$ :

Assumption 2 For all $s \in \mathscr{S}$, $s$ is non-blocking and there exists $s_{0} \in \mathscr{S}_{0}$ such that $s$ is reachable from $s_{0}$.

The assumption above is not restrictive since it is always possible to build from $\mathscr{A}$ an automaton $\mathscr{A}^{\prime}$ satisfying Assumption 2 and such that $L(\mathscr{A})=L\left(\mathscr{A}^{\prime}\right)$. Indeed, $\mathscr{A}^{\prime}$ is obtained firstly by removing all the states not reachable from an initial state and secondly by removing iteratively all blocking states. Remark also that, rigorously speaking, Definition 1 is not the classical definition of nondeterministic finite automaton since we do not define a set of final states, as we consider infinite sequences. Definition 1 actually defines a subclass of nondeterministic Büchi automaton, see [3].

Remark 1 Given $\mathscr{A}$, consider the automaton $(\mathscr{S}, \mathscr{I}, \delta, \mathscr{S})$, i.e. the automaton $\mathscr{A}$ with initial states $\mathscr{S}_{0}=\mathscr{S}$. From Assumption 2, they have the same trajectories and languages, except an initial finite transient required to $s_{0} \in \mathscr{S}_{0}$ to reach any $s \in \mathscr{S}$. In the first part of the paper we will implicitly consider that $\mathscr{S}_{0}=\mathscr{S}$ to analyze the stabilizability and the existence of control Lyapunov functions for the system (1) subject to $L(\mathscr{A})$. The dependence on the set of the automaton initial states will be analyzed in Section 5.

\subsection{Notions of stabilizability}

A control policy $v: \mathbb{R}^{n} \times \mathscr{S} \rightarrow \mathscr{I} \times \mathscr{S}$, is such that

$$
\begin{gathered}
v(x, r)=(i(x, r), m(x, r)) \in \mathscr{I} \times \mathscr{S}, \\
\text { with } \quad m(x, r) \in \delta(r, i(x, r)) .
\end{gathered}
$$

Then, $v$ associates to the state $(x, r)$ a switching mode and an admissible successor of $r$. Note that every mode sequence $\sigma$ generated by $v$ is in $L(\mathscr{A})$, then we denote, with slight abuse of notation, $v \in L(\mathscr{A})$. Moreover we denote with $\left(x_{N}^{v}\left(x_{0}, r_{0}\right), r_{N}^{v}\left(x_{0}, r_{0}\right)\right) \in \mathbb{R}^{n} \times \mathscr{S}$ the state of the system (1) and automaton $\mathscr{A}$ at time $N$ starting from $\left(x_{0}, r_{0}\right)$ by applying the control policy $v$. Thus, $\left(x_{0}^{v}\left(x_{0}, r_{0}\right), r_{0}^{v}\left(x_{0}, r_{0}\right)\right)=\left(x_{0}, r_{0}\right)$. Analogously, given $\sigma \in L(\mathscr{A})$ we denote with $x_{N}^{\sigma}\left(x_{0}\right)$ the state of (1) at time $N$ starting at $x_{0}$ under the switching sequence $\sigma$. To simplify the notation, the dependence of $x_{N}^{v}, r_{N}^{v}$ and $x_{N}^{\sigma}$ on the initial 
conditions may be dropped and we denote $x^{+}=x_{1}^{v}$ and $r^{+}=r_{1}^{v}$. Finally $\sigma \in \mathscr{I}^{N}$ belongs to $L(\mathscr{A})$ if it is the prefix of an element of $L(\mathscr{A})$.

We consider here the following notion of stabilizability:

Definition 2 The system (1) is globally exponentially stabilizable $(G E S)$ relatively to the language $L(\mathscr{A})$ if there are $c \geq 1$ and $\lambda \in[0,1)$ and, for all $x \in \mathbb{R}^{n}$, there exists a switching law $\sigma \in L(\mathscr{A})$, such that

$$
\left\|x_{k}^{\sigma}(x)\right\| \leq c \lambda^{k}\|x\|, \quad \forall k \in \mathbb{N} .
$$

We also give below the definition of exponentially stabilizing control Lyapunov function (ECLF) for switched systems, adapted from that one formulated in [26].

Definition 3 A nonnegative continuous function $V: \mathbb{R}^{n} \times$ $\mathscr{R} \rightarrow \mathbb{R}^{+}$is an exponentially stabilizing control Lyapunov function (ECLF) of system (1) in $\mathscr{R} \subseteq \mathscr{S}$, with $\mathscr{R}$ non-empty, if for every $(x, r) \in \mathbb{R}^{n} \times \mathscr{R}$, we have

(i) $\kappa_{1}\|x\| \leq V(x, r) \leq \kappa_{2}\|x\|$ for some positive constants $\kappa_{1}$ and $\kappa_{2}$;

(ii) $V(x, r)-V\left(x^{+}, r^{+}\right) \geq \kappa_{3}\|x\|$ for some constant $\kappa_{3}>0$ and control policy $v(x, r)$ as in (2) and such that $m(x, r) \in \mathscr{R}$.

The existence of an ECLF implies exponential stabilizability of the system (1) relatively to the language $L(\mathscr{A})$.

Remark 2 Note that it is sufficient that the ECLF as well as the control policy $v$ were defined on a subset of the automaton states $\mathscr{R}$, since every state is assumed in the set of initial states, see Assumption 1 and Remark 1, provided the automaton state is maintained in $\mathscr{R}$ under $v$.

We consider the particular case when the stabilizing switching law is such that the automaton reaches one state $s \in \mathscr{S}$ every $N$ steps at most. We will refer to this class of switching sequences as recurrent.

Definition 4 The automaton trajectory $r^{\sigma}: \mathbb{N} \rightarrow \mathscr{S}$, generating the switching sequence $\sigma \in L(\mathscr{A})$, is ultimately recurrent in $s \in \mathscr{S}$ under $\sigma$ if there exist $m \in \mathbb{N}, N \in \mathbb{N}$ and a sequence $l_{k}: \mathbb{N} \rightarrow \mathbb{N}$ such that $l_{0}=m$ and $r_{l_{k}}^{\sigma}=s$, and $1 \leq l_{k+1}-l_{k} \leq N$ for all $k \in \mathbb{N}$. It is recurrent in $s$ under $\sigma$ if it is ultimately recurrent with $m=0$. The recurrence length is $N$.

The concepts of recurrent stabilizability and recurrent ECLF are defined.

Definition 5 The system (1) is recurrently globally exponentially stabilizable relatively to the language $L(\mathscr{A})$ if there exist $s \in \mathscr{S}, N \in \mathbb{N}, c \geq 1$ and $\lambda \in[0,1)$ such that, for every $x \in \mathbb{R}^{n}$, there exists $\sigma \in L(\mathscr{A})$ and $r^{\sigma}: \mathbb{N} \rightarrow \mathscr{S}$ such that (3) is satisfied and $r^{\sigma}$ is recurrent in s under $\sigma$ with recurrence length $N$.
Control policies that generate recurrent trajectories can be defined.

Definition 6 Given $s \in \mathscr{S}$ and $N \in \mathbb{N}$ positive, a control policy $v^{s}: \mathbb{R}^{n} \rightarrow(\mathscr{I} \times \mathscr{S})^{[1, N]}$ recurrent in $s \in \mathscr{S}$, with recurrence length $N$, is such that

$$
\begin{aligned}
& v_{j}^{s}(x)=\left(i_{j}(x), m_{j}(x)\right) \in \mathscr{I} \times \mathscr{S}, \\
& \text { with } \quad m_{j}(x) \in \delta\left(m_{j-1}(x), i_{j}(x)\right),
\end{aligned}
$$

for all $j \in \mathbb{N}_{p(x)}$, with $p(x)=\left|v^{s}(x)\right|$, and such that $m_{0}(x)=$ $m_{p(x)}(x)=s$.

Thus, a recurrent control policy, related to the automaton state $s$, associates to every system state $x$ a sequence of control actions (composed by switching mode and admissible successor, see (2)) that generates automaton trajectories recurrent in $s$. Note that the switching sequences generated by $v^{s}$ belong to $L(\mathscr{A})$, by construction, and then we write $v^{s} \in L(\mathscr{A})$.

Definition 7 A nonnegative continuous function $V^{s}: \mathbb{R}^{n} \rightarrow$ $\mathbb{R}^{+}$is a recurrent exponentially stabilizing control Lyapunov function of system (1) in $s \in \mathscr{S}$ with recurrence length $N \in \mathbb{N}$ if for every $x \in \mathbb{R}^{n}$, we have

(i) $\kappa_{1}\|x\| \leq V^{s}(x) \leq \kappa_{2}\|x\|$ for some positive constants $\kappa_{1}$ and $\kappa_{2}$;

(ii) $V^{s}(x)-V^{s}\left(x_{p(x)}^{v^{s}}\right) \geq \kappa_{3}\|x\|$ for some constant $\kappa_{3}>0$ and control policy $v^{s}$ recurrent in $s$ with recurrence length $N$.

From Definition 7, a recurrent ECLF is defined only on the system state space and is such that a control policy, recurrent in $s$, exists that makes it decrease every time the automaton state reaches $s$, for every $x \in \mathbb{R}^{n}$. The existence of a recurrent ECLF is sufficient for systems (1) to be GES, as proved below.

Theorem 1 If a recurrent exponentially stabilizing control Lyapunov function in $s \in \mathscr{S}$ exists for system (1), then the system is globally exponentially stabilizable relatively to the language $L(\mathscr{A})$.

Proof: First note that, from the Definition 7, if a recurrent ECLF exists then there is a recurrent control policy $v^{s}$ such that

$$
V^{s}\left(x_{p(x)}^{v^{s}}\right) \leq \mu V^{s}(x) \quad \text { with } \quad \mu=\left(1-\frac{\kappa_{3}}{\kappa_{2}}\right)
$$

for all $x \in \mathbb{R}^{n}$, where $\mu$ is smaller than one and can be made positive by appropriately choosing $\kappa_{3}$, small enough, and $\kappa_{2}$, big enough, if necessary. Since $v^{s}$ generates recurrent trajectories $r^{v^{s}}$, see Definitions 4 and 6 , a sequence of instants $l_{k}$ exists where $r_{l_{k}}^{v^{s}}=s$, with $k \in \mathbb{N}$, such that $1 \leq l_{k+1}-l_{k} \leq N$, 
with recurrence length $N$ and $l_{0}=0$. From (4) it follows

$$
V^{s}\left(x_{l_{k}}^{v^{s}}\right) \leq \mu V^{s}\left(x_{l_{k-1}}^{\nu^{s}}\right) \leq \mu^{k} V^{s}(x)
$$

and then, denoting $L=\max \left\{1, \max _{i \in \mathscr{I}}\left\{\left\|A_{i}\right\|\right\}\right\}$, for every $j \in \mathbb{N}$ positive there exists $k \in \mathbb{N}$ such that $l_{k}<j \leq l_{k+1}$ and

$$
\begin{aligned}
\left\|x_{j}^{v^{s}}\right\| & \leq L^{j-l_{k}}\left\|x_{l_{k}}^{v^{s}}\right\| \leq L^{N}\left\|x_{l_{k}}^{v^{s}}\right\| \leq \frac{L^{N}}{\kappa_{1}} V^{s}\left(x_{l_{k}}^{v^{s}}\right) \\
& \leq \frac{L^{N}}{\kappa_{1}} \mu^{k} V^{s}(x) \leq L^{N} \frac{\kappa_{2}}{\kappa_{1}} \mu^{k}\|x\|
\end{aligned}
$$

since $L \geq 1$. Finally, from $j \leq l_{k+1}$ and $l_{k+1} \leq N+l_{k} \leq$ $(k+1) N$, it follows that $k \geq j / N-1$ and then

$$
\left\|x_{j}^{v^{s}}\right\| \leq L^{N} \frac{\kappa_{2}}{\kappa_{1} \mu} \mu^{j / N}\|x\|
$$

and thus the system (1) is GES.

\section{Necessary and Sufficient Condition with Recurrence}

The main results of the paper, concerning the constructive conditions for the existence of an ECLF and the relations with recurrent GES, are presented in this section.

\subsection{Geometric condition}

The C-sets, i.e. compact, convex sets containing the origin in their interior, and the induced gauge functions are widely employed for robust stability and stabilizability of parametric uncertain linear systems, [4,5]. In this paper we employ analogous geometrical concepts.

Definition 8 A set $\Omega \subseteq \mathbb{R}^{n}$ is a $C^{*}$-set if it is the union of a finite number of $C$-sets. The gauge function of a $C^{*}$-set $\Omega \subseteq \mathbb{R}^{n}$ is $\Psi_{\Omega}(x)=\min _{\alpha \geq 0}\{\alpha \in \mathbb{R}: x \in \alpha \Omega\}$.

Notice that every $\mathrm{C}^{*}$-set is star-convex, i.e. there is $z \in \Omega$ such that every convex combination of $x$ and $z$ belongs to $\Omega$ for all $x \in \Omega$, but the converse is not true in general. Some basic properties of the $\mathrm{C}^{*}$-sets and their gauge functions are listed below, see also [19].

Property 1 Every $C$-set is a $C^{*}$-set. Given a $C^{*}$-set $\Omega \subseteq$ $\mathbb{R}^{n}$, we have that $\alpha \Omega \subseteq \Omega$ for all $\alpha \in[0,1]$, and the gauge function $\Psi_{\Omega}(x)$ is continuous; homogeneous of degree one, i.e. $\Psi_{\Omega}(\alpha x)=\alpha \Psi_{\Omega}(x)$ for all $\alpha \geq 0$ and $x \in \mathbb{R}^{n}$; positive definite; defined on $\mathbb{R}^{n}$ and radially unbounded. For every family of $C^{*}$-sets $\Omega_{i}$ with $i \in \mathbb{N}_{I}$, also the sets $\underline{\Omega}=\bigcup_{i \in I} \Omega_{i}$ and $\bar{\Omega}=\bigcap_{i \in I} \Omega_{i}$ are $C^{*}$-sets and $\Psi_{\underline{\Omega}}(x)=\min _{i \in \mathbb{N}_{I}} \Psi_{\Omega_{i}}(x)$ and $\Psi_{\bar{\Omega}}(x)=\max _{i \in \mathbb{N}_{I}} \Psi_{\Omega_{i}}(x)$. Moreover $\Psi_{\mathscr{B}^{n}}(x)=\|x\|$ and $\Omega_{i} \subseteq \Omega_{j}$ if and only if $\Psi_{\Omega_{j}}(x) \leq \Psi_{\Omega_{i}}(x)$ for all $x \in \mathbb{R}^{n}$. Finally, for every nonsingular matrix $A \in \mathbb{R}^{n \times n}$ and $C^{*}$-set $\Omega \subseteq \mathbb{R}^{n}$, also $A \Omega$ is a $C^{*}$-set in $\mathbb{R}^{n}$ and $\Psi_{A \Omega}(x)=\Psi_{\Omega}\left(A^{-1} x\right)$.
The gauge functions induced by $\mathrm{C}$-sets have been used in literature as Lyapunov functions candidates, for linear parametric uncertain systems, $[17,4]$, and switched systems with arbitrary switching, [16]. On the other hand, the gauge functions of $\mathrm{C}^{*}$-sets are proved to be a universal class of Lyapunov functions for switched systems with switching control law, see $[9,8]$. We will be searching, then, for contractive $\mathrm{C}^{*}$-sets such that the related gauge functions could result to be recurrent ECLF for the switched systems subject to the language constraints induced by $\mathscr{A}$.

For every set $\Omega \subseteq \mathbb{R}^{n}$, state $s \in \mathscr{S}$ and mode $i \in \mathscr{I}$, define the one-step operator for the switched system (1) whose switching law is specified by $\mathscr{A}$ as

$$
Q_{i}^{s}(\Omega)=\left\{(x, r) \in \mathbb{R}^{n} \times \mathscr{S}: A_{i} x \in \Omega, \quad s \in \delta(r, i)\right\} .
$$

Namely, given a set $\Omega$, a state of the automaton $s$ and a mode $i$, the operator $Q_{i}^{s}(\Omega)$ gives

$$
Q_{i}^{s}(\Omega)=\bigcup_{r \in \gamma(s, i)}\left(A_{i}^{-1} \Omega \times r\right)
$$

where $\gamma(s, i)$ is the backward operator for the automaton, i.e. $\gamma(s, i)=\{r \in \mathscr{S}: s \in \delta(r, i)\}$. Thus, intuitively, $Q_{i}^{s}(\Omega)$ is the preimage through the mode $i \in \mathscr{I}$, in the space $\mathbb{R}^{n} \times \mathscr{S}$, of the set $\Omega \times s$. Clearly, if $s \notin \delta(r, i)$ for all $r \in \mathscr{S}$, then $Q_{i}^{s}(\Omega)=\emptyset$. Moreover define:

$$
Q^{s}(\Omega)=\bigcup_{i \in \mathscr{I}} Q_{i}^{s}(\Omega)=\bigcup_{i \in \mathscr{I}} \bigcup_{r \in \gamma(s, i)}\left(A_{i}^{-1} \Omega \times r\right)
$$

that is the set of the preimages of the set $\Omega \times s$ through all the modes $i \in \mathscr{I}$. Consider the Algorithm 1 below (which is a semi-algorithm, to be exact), where index $s$ denotes the initial automaton state.

Algorithm 1 Computation of a contractive $C^{*}$-set for the system (1), satisfying Assumption 1, recurrent in s.

- Initialization: given the $C^{*}$-set $\Omega_{0} \subseteq \mathbb{R}^{n}$ and a state $s \in \mathscr{S}$, define $\Lambda_{0}^{s}=\Omega_{0} \times s$ and $k=0$;

- Iteration for $k \geq 0$ :

$$
\begin{aligned}
& \Lambda_{k+1}^{s}=\bigcup_{(\Omega \times r) \subseteq \Lambda_{k}^{s}} Q^{r}(\Omega), \\
& \Omega_{k+1}^{s}=\left\{x \in \mathbb{R}^{n}:(x, s) \in \Lambda_{k+1}^{s}\right\},
\end{aligned}
$$

- Stop if $\Omega_{0} \subseteq \operatorname{int}\left(\bigcup_{j \in \mathbb{N}_{k+1}} \Omega_{j}^{s}\right)$; denote $N^{s}=k+1$ and

$$
\Omega^{s}=\bigcup_{j \in \mathbb{N}_{N^{s}}} \Omega_{j}^{s}
$$


If Algorithm 1 terminates, the following sets

$$
\begin{aligned}
& \Omega_{j}^{r, s}=\left\{x \in \mathbb{R}^{n}:(x, r) \in \Lambda_{j}^{s}\right\}, \quad \forall j \in \mathbb{N}_{N^{s}}, \\
& \Omega^{r, s}=\bigcup_{j \in \mathbb{N}_{N^{s}}} \Omega_{j}^{r, s},
\end{aligned}
$$

are defined for all $r \in \mathscr{S}$. Note that $\Omega_{j}^{s, s}=\Omega_{j}^{s}$.

The geometrical interpretations of the sets $\Lambda_{j}^{s}$ and $\Omega_{j}^{r, s}$, with $r \in \mathscr{S}$, follow, their dependence on $\Omega_{0}$ is left implicit. Given $\Lambda_{k}^{s} \subseteq \mathbb{R}^{n} \times \mathscr{S}$, the points in $\mathbb{R}^{n} \times \mathscr{S}$ that can be steered in one step in $\Lambda_{k}^{s}$, by means of an admissible mode, are the set $\Lambda_{k+1}^{s}$. Thus, $\Lambda_{j}^{s}$ is the set of $(x, r) \in \mathbb{R}^{n} \times \mathscr{S}$ that can be steered in $\Omega_{0} \times s$ by means of admissible sequences of modes of length $j$. The set $\Omega_{j}^{r, s}$ is, then, the set of states $x \in \mathbb{R}^{n}$ such that, if the automaton state is $r$, a switching sequence exists that steers $x$ in $\Omega_{0}$ in $j$ steps, with the automaton state reaching $s$.

In particular $\Omega_{j}^{r, s}$ is the union of the preimages of sets $\Omega_{j-1}^{t, s}$, for some $t \in \mathscr{S}$ and through admissible modes $i$. In fact, an alternative, constructive, definition of $\Omega_{j}^{r, s}$ is obtained by defining

$$
\begin{aligned}
\Gamma_{j}(r, s)= & \left\{(i, t) \in(\mathscr{I} \times \mathscr{S}): \exists(\sigma, m) \in(\mathscr{S} \times \mathscr{I})^{j}\right. \text { s.t. } \\
& (i, t)=\left(\sigma_{1}, m_{1}\right), m_{1} \in \delta\left(r, \sigma_{1}\right), m_{|\sigma|}=s, \\
& \left.m_{k+1} \in \delta\left(m_{k}, \sigma_{k+1}\right) \forall k \in \mathbb{N}_{j-1}\right\}
\end{aligned}
$$

for $s, r \in \mathscr{S}$ and $j \in \mathbb{N}_{N^{s}}$. In practice, $\Gamma_{j}(r, s)$ characterizes the first element of all the admissible switching sequences driving the system state from $\Omega^{r, s}$ to $\Omega_{0}$ and the automaton state from $r$ to $s$ in $j$ steps. Then, for every $r \in \mathscr{S}$ and $j \in \mathbb{N}_{N^{s}}$, the sets $\Omega_{j}^{r, s}$ can be recursively determined as

$$
\Omega_{j}^{r, s}=\bigcup_{(i, t) \in \Gamma_{j}(r, s)} A_{i}^{-1} \Omega_{j-1}^{t, s}
$$

starting with $\Omega_{0}^{s, s}=\Omega_{0}$.

Lemma 1 If Assumption 1 holds, the sets $\Omega_{j}^{r, s}$ are $C^{*}$-sets or empty, for all $j \in \mathbb{N}_{N^{s}}$ and $r \in \mathscr{S}$.

Proof: The result comes directly from the properties of $\mathrm{C}^{*}$-sets, see Property 1 .

A first main result, providing a constructive method for determining whether the system (1) can be stabilized by means of a switching sequence in $L(\mathscr{A})$, is now presented. The Assumption 1 is supposed to hold, the case of singular matrices $A_{i}$ is treated in Section 3.3.

Theorem 2 Let Assumption 1 hold. There exists a recurrent exponential control Lyapunov function in $s \in \mathscr{S}$ if and only if Algorithm 1 terminates in finite time for the automaton state $s$.
Proof: First we prove sufficiency, that is the fact that the finite termination of the algorithm implies the existence of a recurrent ECLF in $s$. Suppose that the algorithm terminates with finite $N^{s} \in \mathbb{N}$. From the geometrical meaning, $\Omega^{s}$ is the set of $x \in \mathbb{R}^{n}$ that can be driven to $\Omega_{0}$, with the automaton initial and final states equal to $s$, by means of an admissible switching sequence of length $p(x)$ smaller than or equal to $N^{s}$, that is through a recurrent control policy $v^{s}$ as in Definition 6. Moreover, since $\Omega_{0}$ and $\Omega^{s}$ are $C^{*}$-sets, then there exists $\rho \in(0,1)$ such that

$$
\Omega_{0} \subseteq \rho \Omega^{s} \subseteq \Omega^{s}
$$

from $\Omega_{0} \subseteq \operatorname{int}\left(\Omega^{s}\right)$. Consider the function

$$
V^{s}(x)=\Psi_{\Omega^{s}}(x)
$$

that is continuous, positive definite and homogeneous of order one, from Property 1 and Lemma 1, and then satisfies $(i)$ of Definition 7. From the properties of the gauge function we have that $x \in \partial\left(\Psi_{\Omega^{s}}(x) \Omega^{s}\right)$, which implies $x_{p(x)}^{v^{s}} \in \Psi_{\Omega^{s}}(x) \Omega_{0}$, from homogeneity. From (12), it follows $x_{p(x)}^{v^{s}} \in \Psi_{\Omega^{s}}(x) \rho \Omega^{s}$ and then

$$
V^{s}\left(x_{p(x)}^{v^{s}}\right)=\Psi_{\Omega^{s}}\left(x_{p(x)}^{v^{s}}\right) \leq \rho \Psi_{\Omega^{s}}(x)=\rho V^{s}(x)
$$

that implies satisfaction of $(i i)$ in Definition 7. Then, $V^{s}(x)$ defined in (13) is a recurrent ECLF.

Concerning necessity, suppose that a recurrent ECLF in $s \in \mathscr{S}$ exists. This implies that the system is exponentially stabilizable under a control policy $v^{s}$ recurrent in $s$, from Theorem 1. Then $v^{s}$ generates trajectories $r^{v^{s}}$ recurrent in $s$, with recurrence length $N \in \mathbb{N}$, and such that $\left\|x_{k}^{\nu^{s}}\right\| \leq c \lambda^{k}\|x\|$ for all $x \in \mathbb{R}^{n}$, with $\lambda \in[0,1)$. Given the initial $\mathrm{C}^{*}$-set $\Omega_{0}$, fix $\tau \in(0,1)$ and define

$$
\begin{aligned}
& \alpha_{m}^{s}=\max _{a \geq 0}\left\{\alpha \in \mathbb{R}: \alpha \mathscr{B}^{n} \subseteq \Omega_{0}\right\}, \\
& \alpha_{M}^{s}=\min _{a \geq 0}\left\{\alpha \in \mathbb{R}: \Omega_{0} \subseteq \alpha \tau \mathscr{B}^{n}\right\},
\end{aligned}
$$

and $\beta=\alpha_{m}^{s} / \alpha_{M}^{s}$. Note that $\beta \in(0,1)$. Defining also $K=$ $\min _{k \in \mathbb{N}}\left\{k \geq 1: c \lambda^{k} \leq \beta\right\}$, it follows that for all $x \in \mathbb{R}^{n}$ one has

$$
\left\|x_{K+k}^{\nu^{s}}\right\| \leq c \lambda^{K+k}\|x\| \leq \beta\|x\|, \quad \forall k \in \mathbb{N}_{N}
$$

with $r_{0}=s$ and $r_{K+k}^{v^{s}}=s$ for at least a $k \in \mathbb{N}_{N}$, from recurrence. This implies that

$$
\begin{aligned}
& \forall\left(x_{0}, r_{0}\right) \in\left(\beta^{-1} \mathscr{B}^{n}\right) \times s \Rightarrow \\
& \exists k \in \mathbb{N}_{N} \text { s.t. }\left(x_{K+k}^{v^{s}}, r_{K+k}^{v^{s}}\right) \in \mathscr{B}^{n} \times s,
\end{aligned}
$$


that is equivalent, from homogeneity, to

$$
\begin{aligned}
& \forall\left(x_{0}, r_{0}\right) \in\left(\alpha_{M}^{s} \mathscr{B}^{n}\right) \times s \Rightarrow \\
& \exists k \in \mathbb{N}_{N} \text { s.t. }\left(x_{K+k}^{v^{s}}, r_{K+k}^{v^{s}}\right) \in\left(\alpha_{m}^{s} \mathscr{B}^{n}\right) \times s .
\end{aligned}
$$

Initializing Algorithm 1 with $\Omega_{0}$, which is such that $\alpha_{m}^{s} \mathscr{B}^{n} \subseteq$ $\Omega_{0}$, and iterating $K+N$ steps one obtains a set that contains all the points $(x, r)$ that can be steered in $\alpha_{m}^{s} \mathscr{B}^{n} \times s$ in $K+N$ steps or less. Therefore, from (14), we have

$$
\left(\alpha_{M}^{s} \mathscr{B}^{n}\right) \times s \subseteq \bigcup_{j \in \mathbb{N}_{K+N}}\left(\Omega_{j}^{s, s} \times s\right),
$$

which implies, from $\Omega_{0} \subseteq \tau \alpha_{M}^{s} \mathscr{B}^{n} \subseteq \operatorname{int}\left(\alpha_{M}^{s} \mathscr{B}^{n}\right)$, that the stop condition (8) is satisfied.

Theorem 2 establishes the equivalence between the existence of a recurrent ECLF and the finite termination of Algorithm 1 . The relations with recurrent stabilizability and with general ECLFs are dealt with in the next section.

Remark 3 If the Algorithm 1 terminates in finite time then every initial state $x \in \mathbb{R}^{n}$ can be exponentially stabilized. In fact, by Assumption 2 and Remark 1, the automaton state $s$ can be considered as an initial state and the recurrent ECLF, being the gauge function of a $C^{*}$-set, is defined on the whole $\mathbb{R}^{n}$, see Property 1.

\subsection{Recurrent stabilizability and existence of ECLF}

The existence of a recurrent ECLF is proved in Theorem 1 to be sufficient for stabilizability and can be obtained in finite time if it exists, see Theorem 2. Recurrent stabilizability can be proved to be also sufficient for the existence of a recurrent ECLF.

Proposition 1 Let Assumption 1 hold. The system (1) is recurrently globally exponentially stabilizable if and only if there exists a recurrent ECLF.

Proof: The fact that the existence of a recurrent ECLF implies recurrent GES follows directly from Theorem 1 . We have to prove that if system (1) is recurrently GES then a recurrent ECLF exists. From recurrent GES there exist $s \in \mathscr{S}, N \in \mathbb{N}, c \geq 1$ and $\lambda \in[0,1)$ such that, given $\mu \in$ $[0,1)$, for every $\left(x_{0}, r_{0}\right) \in \mathscr{B}^{n} \times s$ there are $\sigma=\sigma\left(x_{0}, r_{0}\right) \in$ $L(\mathscr{A})$ and $k=k\left(x_{0}, r_{0}\right) \in \mathbb{N}$, with $\log _{\lambda}(\mu / c) \leq k\left(x_{0}, r_{0}\right) \leq$ $\log _{\lambda}(\mu / c)+N$, such that $\left(x_{k}^{\sigma}, r_{k}^{\sigma}\right) \in\left(\mu \mathscr{B}^{n}\right) \times s$, since by construction $c \lambda^{k\left(x_{0}, r_{0}\right)} \leq \mu$. Thus taking $K=\max \left\{k\left(x_{0}, r_{0}\right)\right.$ : $\left.x_{0} \in \mathscr{B}^{n}, r_{0}=s\right\}$, the Algorithm 1 with $\Omega_{0}=\mu \mathscr{B}^{n}$ terminates after $K$ steps at most. This implies the existence of a recurrent ECLF, from Theorem 2.

Then, the finite termination of Algorithm 1 is equivalent to the existence of a recurrent ECLF and to recurrent GES. We analyze hereafter the generality of recurrent ECLF, that is their relation with general ECLF defined in Definition 3.
Proposition 2 Let Assumption 1 hold. If Algorithm 1 terminates in finite time for a state $s \in \mathscr{S}$ then there exist a non-empty set $\mathscr{R} \subseteq \mathscr{S}$ and an exponential control Lyapunov function in $\mathscr{R}$.

Proof: Given $\rho$ as in (12), define $\mu=\rho^{1 / N^{s}}$ and notice that $\mu \in(0,1)$. The proof is analogous to the one in [8] with

$$
V(x, r)=\min _{j \in \mathbb{N}_{N^{s}}} \mu^{-j} \Psi_{\Omega_{j}^{r, s}}(x)
$$

continuous positive definite function. Defining

$$
j(x, r)=\arg \min _{j \in \mathbb{N}_{N^{s}}} \mu^{-j} \Psi_{\Omega_{j}^{r, s}}(x)
$$

it follows that $V(x, r)=\mu^{-j(x, r)} \Psi_{\Omega_{j(x, r)}^{r, s}}(x)$. Since $x \in$ $\partial\left(\Psi_{\Omega}(x) \Omega\right)$ for every $C^{*}$-set $\Omega$, then $x \in \partial\left(V(x, r) \mu^{j(x, r)} \Omega_{j(x, r)}^{r, s}\right)$ which implies that there exists $(i, t) \in \Gamma_{j(r, s)}$ such that

$$
A_{i} x \in V(x, r) \mu^{j(x, r)} \Omega_{j(x, r)-1}^{t, s}
$$

from (10) and (11) and then the following control policy

$v(x, r) \in\left\{(i, t) \in \Gamma_{j(x, r)}(r, s): A_{i} x \in V(x, r) \mu^{j(x, r)} \Omega_{j(x, r)-1}^{t, s}\right\}$

is defined. If $j(x, r) \geq 2$, then with $(i, t)=v(x, r)$ we have

$$
\begin{aligned}
V\left(A_{i} x, t\right) & =\min _{j \in \mathbb{N}_{N^{s}}} \mu^{-j} \Psi_{\Omega_{j}^{t, s}}\left(A_{i} x\right) \leq \mu^{-j(x, r)+1} \Psi_{\Omega_{j(x, r)-1}^{t, s}}\left(A_{i} x\right) \\
& \leq \mu^{-j(x, r)+1} \mu^{j(x, r)} V(x, r)=\mu V(x, r)
\end{aligned}
$$

from (17). For $j(x, r)=1$ thus $t=s$ and $A_{i} x \in V(x, r) \mu \Omega_{0}$ for $(i, s)=v(x, r)$, from (17), and therefore, from $\Psi_{\Omega^{s}}(x) \leq$ $\rho \Psi_{\Omega_{0}}(x)$, it follows

$$
\begin{aligned}
V\left(A_{i} x, s\right) & =\mu^{-j\left(A_{i} x, s\right)} \Psi_{\Omega_{j\left(A_{i} x, s\right)}^{s, s}}\left(A_{i} x\right)=\mu^{-j\left(A_{i} x, s\right)} \Psi_{\Omega^{s}}\left(A_{i} x\right) \\
& \leq \mu^{-N^{s}} \Psi_{\Omega^{s}}\left(A_{i} x\right) \leq \mu^{-N^{s}} \rho \Psi_{\Omega_{0}}\left(A_{i} x\right)=\mu V(x, r),
\end{aligned}
$$

since $\mu^{N^{s}}=\rho$. Thus, $V(x, r)$ is an ECLF on $\mathscr{R}$ given by the automaton states $r$ for which $\Omega^{r, s} \neq \emptyset$.

Note that the control policy (18) does not ensure, in general, recurrence of the automaton trajectories.

Above we proved that the existence of a recurrent ECLF, equivalent to the finite termination of Algorithm 1, is sufficient for the existence of an ECLF. Hereafter we analyze several aspects on necessity for stabilizability and on the relations with general ECLF.

The first issue is to prove, or exclude, that the recurrent ECLF are not conservative with respect to the generic ECLF. That is, we want to prove that the existence of a recurrent 
ECLF is necessary, as well as sufficient, for the existence of an ECLF as defined in Definition 3. For this, some concept related to the theory of graphs are to be introduced.

With the automaton $\mathscr{A}$, it is possible to associate the finite directed graph or digraph $\mathscr{G}=(\mathscr{V}, \mathscr{E})$, for which the set of nodes is the finite set of states $\mathscr{V}=\mathscr{S}$ and the set of edges is related to the transitions map $\delta: \mathscr{E}=\left\{(s, r) \in \mathscr{S}^{2}: \exists \ell \in\right.$ $\mathscr{I}, r \in \delta(s, \ell)\}$.

Some definitions and properties related to the digraph $\mathscr{G}$ and its Strongly Connected Components (SCC) are given in Appendix A. Using Proposition 5 in appendix, the equivalence between the existence of ECLF and recurrent ECLF can be proved.

Theorem 3 Let Assumption 1 hold. There exists an exponential control Lyapunov function for the system (1) in $\mathscr{R} \subseteq$ $\mathscr{S}$, non-empty, if and only if there exists a recurrent exponential control Lyapunov function in $s \in \mathscr{S}$.

Proof: Proposition 2 proves sufficiency. We prove necessity, that is the fact that the existence of an ECLF in $\mathscr{R}$ implies the existence of a recurrent one, in $s \in \mathscr{S}$. Suppose that $V(x, r)$ is an ECLF defined in $\mathbb{R}^{n} \times \mathscr{R}$. From Proposition 5 in appendix, the set $\mathscr{R}$ must contain at least one node belonging to a nontrivial SCC. Denote with $\overline{\mathscr{C}}$ one nontrivial SCC such that there is not another SCC $\mathscr{C}_{i}$ containing a node in $\mathscr{R}$ and $\overline{\mathscr{C}} \succeq \mathscr{C}_{i} . \overline{\mathscr{C}}$ exists since the condensation is acyclic. Select one node $\bar{s} \in \overline{\mathscr{C}} \cap \mathscr{R}$ and denote $\bar{p}=|\overline{\mathscr{C}}|$. From the definition of $\overline{\mathscr{C}}$ all the trajectories starting with automaton state in $\bar{s}$ exponentially converge to the origin while the automaton state remains in $\overline{\mathscr{C}}$ under the control policy (2). Moreover, since $\overline{\mathscr{C}}$ is a SCC, every node in $\overline{\mathscr{C}}$ is strongly connected to $\bar{s}$. For every $r \in \overline{\mathscr{C}}$ with $r \neq \bar{s}$ define

$$
\begin{gathered}
\sigma^{r, \bar{s}}=\arg \min _{\sigma \in \mathscr{I}[1: \bar{p}-1]}\left\{j=|\sigma|: \exists m \in \overline{\mathscr{C}}^{j+1} \text { s.t. } m_{1}=r,\right. \\
\left.m_{j+1}=s, \quad m_{k+1} \in \delta\left(m_{k}, \sigma_{k}\right) \quad \forall k \in \mathbb{N}_{j}, \quad \sigma \in L(\mathscr{A})\right\}
\end{gathered}
$$

that is the set of switching sequences in $L(\mathscr{A})$ that generate the shortest paths in $\overline{\mathscr{C}}$ between $r$ and $\bar{s}$, and

$$
\rho^{r, \bar{s}}=\max _{\sigma \in \sigma^{r, \bar{s}}}\left\|\mathbb{A}_{\sigma}\right\|, \quad \rho=\max _{r \in \overline{\mathscr{C}}, r \neq \bar{s}} \rho^{r, \bar{s}},
$$

where $\mathbb{A}_{\sigma}=A_{\sigma_{N}} \ldots A_{\sigma_{1}}$ for all $\sigma \in \mathscr{I}^{N}$. Thus, every $(x, r) \in$ $\mathscr{B}^{n} \times \overline{\mathscr{C}}$ can be steered in $\rho \mathscr{B}^{n} \times \bar{s}$ through an admissible sequence $\sigma(x, r) \in L(\mathscr{A})$. From the existence of the ECLF, defined in $\bar{s}$ by hypothesis, every $(x, r) \in \mathscr{B}^{n} \times \bar{s}$ can be driven in finite time in $\frac{\varepsilon}{\rho} \mathscr{B}^{n} \times \overline{\mathscr{C}}$, for every $\varepsilon \in(0,1)$, by means of the control policy $v(x, r)$ related to the ECLF. Then, from what said above, $(x, r) \in \mathscr{B}^{n} \times \bar{s}$ can be steered in $\varepsilon \mathscr{B}^{n} \times \bar{s}$ by a sequence in $L(\mathscr{A})$, which implies that the Algorithm 1 initialized with $s=\bar{s}$ and $\Omega_{0}=\varepsilon \mathscr{B}^{n}$ would end in finite time. Hence, from Theorem 2, a recurrent ECLF exists.
Above we proved that the existence of a recurrent ECLF defined in an automaton state $s$ is necessary and sufficient for the existence of an ECLF. This means that the attention could be restricted to recurrent ECLFs. Moreover, its existence has been proved to be equivalent to the finite termination of Algorithm 1, leading to a constructive necessary and sufficient condition for the existence of an ECLF.

\subsection{Nonsingular matrices}

As noted above, imposing the nonsingularity of matrices $A_{i}$ is not necessary for the definition of Algorithm 1. Indeed, the preimage of a set $A^{-1} \Omega$, is well-defined, even if $A$ is singular. Then the operator $Q_{i}^{S}(\Omega)$, as in (5) and (6), is welldefined also for singular matrices, although, in this case, it provides unbounded closed sets instead of compact ones. Thus, if some $A_{i}$ are singular, some of the sets $\Omega_{j}^{s}$ might be unbounded, but the stop condition would still imply that the states in $\Omega^{s}$ can be steered in $\Omega_{0}$ in $N^{s}$ steps at most. The main issue is that, in case of singular matrices, the sets $\Omega_{j}^{r, s}$ might be unbounded, for some $r \in \mathscr{S}$, from definition (9), see the example below.

Example 1 Consider the system (1) with

$$
A_{1}=\left[\begin{array}{cc}
0.5 & 0 \\
0 & 0.5
\end{array}\right], \quad A_{2}=\left[\begin{array}{ll}
1 & 0 \\
0 & 0
\end{array}\right],
$$

and constraints given by the automaton of Figure 1.

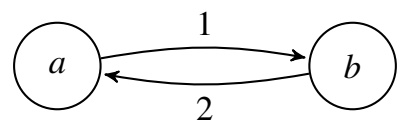

Fig. 1. Automaton of Example 1.

Starting with $\Omega_{0}=\mathscr{B}^{2}$ and $s=a$, Algorithm 1 ends in two steps with $\Omega_{1}^{a}=\emptyset$ and $\Omega_{2}^{a}=\left\{x \in \mathbb{R}^{2}:\left|x_{1}\right| \leq 2\right\}$, unbounded. Moreover, from (9), we have $\Omega_{1}^{b, a}=\left\{x \in \mathbb{R}^{2}:\left|x_{1}\right| \leq 1\right\}$, unbounded.

Roughly speaking, the unbounded sets are due to the preimage operator employed in generating the sets $\Omega_{j}^{r, s}$. Since the Lyapunov functions considered must be positive for every $x \neq 0$, see Definitions 3 and 7 , the gauge function of unbounded sets should not be employed. Notice that boundedness has been imposed above through Definition 8 of $\mathrm{C}^{*}$-sets and Lemma 1.

Lyapunov functions that are positive for all $x \in \mathbb{R}^{n}$, except in the origin, can be obtained by modifying the definition of sets $\Omega_{j}^{r, s}$, for all $r \in \mathscr{S}$. If the Algorithm 1 terminates after $N^{s}$ steps with some unbounded $\Omega_{j}^{r, s}$, an alternative sequence of bounded sets can be defined to induce control Lyapunov functions analogous to those valid under Assumption 1. 
Starting from $j=1$ and $\bar{\Omega}_{0}^{s, s}=\Omega_{0}$, bounded since $\mathrm{C}^{*}$-set, define iteratively, for every $r \in \mathscr{S}$, the set

$$
\bar{\Omega}_{j}^{r, s}=\bigcup_{(i, t) \in \Gamma_{j}(r, s)} A_{i}^{-1} \bar{\Omega}_{j-1}^{t, s} \cap \rho_{r} \mathscr{B}^{n}
$$

which is bounded, for every $\rho_{r}>0$. The dependence on the value of $\rho_{r}$ are left implicit for notational reasons. Clearly $\bar{\Omega}_{j}^{r, s} \subseteq \Omega_{j}^{r, s}$ for all $j \in \mathbb{N}_{N^{s}}$ and $r \in \mathscr{S}$.

Lemma 2 For every $j \in \mathbb{N}_{N^{s}}$ and every $r \in \mathscr{S}$, the sets $\bar{\Omega}_{j}^{r, s}$ given by (20) with initialization $\bar{\Omega}_{0}^{s, s}=\Omega_{0}$ are such that if $x \in \bar{\Omega}_{j}^{r, s}$ then there are $i \in \mathscr{I}$ and $t \in \mathscr{S}$ such that $t \in \delta(r, i)$ and $A_{i} x \in \bar{\Omega}_{j-1}^{t, s}$.

Proof: Since $x \in \bar{\Omega}_{j}^{r, s}$ then there are $i \in \mathscr{I}$ and $t \in$ $\mathscr{S}$ such that $x \in A_{i}^{-1} \bar{\Omega}_{j-1}^{t, s} \cap \rho_{r} \mathscr{B}^{n}$ and $t \in \delta(r, i)$, from the definition (10). Thus, $x \in A_{i}^{-1} \bar{\Omega}_{j-1}^{t, s}$ which means that $A_{i} x \in$ $\bar{\Omega}_{j-1}^{t, s}$.

Lemma 2 implies that, by construction, every element of $\bar{\Omega}_{j}^{r, s}$ can be steered in $\Omega_{0}$ in $j$ steps by an admissible switching sequence driving $r$ to $s$ and then

$$
\bar{\Omega}^{r, s}=\bigcup_{j \in \mathbb{N}_{N^{s}}} \bar{\Omega}_{j}^{r, s}
$$

is a set of states that can reach $\Omega_{0}$ in $N^{s}$ steps at most through a switching sequence generating an automaton trajectory starting in $r$ and ending in $s$, for all $r \in \mathscr{S}$. Thus, if $\Omega_{0} \subseteq \operatorname{int}\left(\bar{\Omega}^{s, s}\right)$ then the gauge functions of $\bar{\Omega}_{j}^{s, s}$ and $\bar{\Omega}_{j}^{r, s}$ can be used to determine a recurrent ECLF, as showed in Theorem 2, and an ECLF, as in Proposition 2, the proofs would be substantially the same as for the case of nonsingular matrices.

What is left to prove is the fact that, if Algorithm 1 terminates, then $\Omega_{0} \subseteq \operatorname{int}\left(\bar{\Omega}^{s, s}\right)$ holds for sufficiently big values of $\rho_{r}$, with $r \in \mathscr{S}$.

Proposition 3 Suppose that Algorithm 1 terminates in finite time. Then, for every $\rho>0$, there are $\rho_{r}>0$ with $r \in \mathscr{S}$ such that

$$
\Omega^{s, s} \cap \rho \mathscr{B}^{n} \subseteq \bar{\Omega}^{s, s},
$$

where $\bar{\Omega}^{s, s}$ is recursively determined through (20) and (21) with $\bar{\Omega}_{0}^{s, s}=\Omega_{0}$.

Proof: We proceed by contradiction, supposing that there is $x \in \Omega^{s, s} \cap \rho \mathscr{B}^{n}$ that does not belong to $\bar{\Omega}^{s, s}$ for every finite $\rho_{r}$ with $r \in \mathscr{S}$. From the definition of $\Omega^{s, s}$, there exists an admissible switching sequence $\sigma$, generating an automaton trajectory of length $p \leq N^{s}$, denoted $r^{\sigma}$, starting and terminating in $s$ and such that $x_{p}^{\sigma}(x) \in \Omega_{0}$ and then

$$
\left(\sigma_{p-j+1}, r_{p-j+1}^{\sigma}\right) \in \Gamma_{j}\left(r_{p-j}^{\sigma}, s\right), \quad \forall j \in \mathbb{N}_{p}
$$

from (10). Take $\rho_{r} \in \mathbb{R}$ such that

$$
\rho_{r} \geq \max \left\{\max _{j \in \mathbb{N}_{p}}\left\|x_{j}^{\sigma}(x)\right\|,\|x\|\right\},
$$

for all $r \in \mathscr{S}$. By definition, $\sigma$ and $r^{\sigma}$ are such that $r_{p}^{\sigma}=s$ and $x_{p}^{\sigma}(x) \in \Omega_{0}=\bar{\Omega}_{0}^{s, s}=\bar{\Omega}_{0}^{r_{p}^{\sigma}, s}$. Therefore, $x_{p-1}^{\sigma}(x) \in A_{\sigma_{p}}^{-1} \bar{\Omega}_{0}^{r_{p}^{\sigma}, s}$ and $x_{p-1}^{\sigma}(x) \in \rho_{r} \mathscr{B}^{n}$, from (24), and then $x_{p-1}^{\sigma}(x) \in \bar{\Omega}_{1}^{r_{p-1}^{\sigma}, s}$, from (20) and (23) with $j=1, r=r_{p-1}^{\sigma}, t=r_{p}^{\sigma}=s$ and $i=\sigma_{p}$. Analogously, $x_{p-2}^{\sigma}(x) \in A_{\sigma_{p-1}}^{-1} \bar{\Omega}_{1}^{r_{p-1}^{\sigma}, s} \cap \rho_{r} \mathscr{B}^{n}$ and then $x_{p-2}^{\sigma}(x) \in \bar{\Omega}_{2}^{r_{p-2}^{\sigma}, s}$. Recursively repeating the reasonings above, we have that $x_{p-j}^{\sigma}(x) \in \bar{\Omega}_{j}^{r_{p-j}^{\sigma}, s}$, for every $j \in \mathbb{N}_{p-1}$, and $x \in \bar{\Omega}_{p}^{r_{0}^{\sigma}, s}=\bar{\Omega}_{p}^{s, s}$ which contradicts the hypothesis that $x$ does not belong to $\bar{\Omega}^{s, s}$.

Proposition 3 implies that, by fixing $\rho$ such that $\Omega_{0} \subseteq$ $\operatorname{int}\left(\rho \mathscr{B}^{n}\right)$ and by appropriately choosing the bounding parameters $\rho_{r}$ with $r \in \mathscr{S}$, the condition $\Omega_{0} \subseteq \operatorname{int}\left(\bar{\Omega}^{s, s}\right)$ is satisfied, since finite termination of Algorithm 1 ensures $\Omega_{0} \subseteq \operatorname{int}\left(\Omega^{s, s}\right)$.

Summarizing, even if Assumption 1 does not hold and Algorithm 1 terminates with $\Omega^{r, s}$ unbounded, $\mathrm{C}^{*}$-sets $\bar{\Omega}^{r, s}$ exist that can be used to determine recurrent ECLFs and ECLFs, as in the proof of Theorem 3 and Proposition 2.

\section{Stabilizability without ECLF}

We are now concerned with the problem of analyzing whether the existence of an ECLF is necessary, as well as sufficient, for exponential stabilizability of a switched system (1) subject to language constraints. A counterexample is given which proves that necessity does not hold.

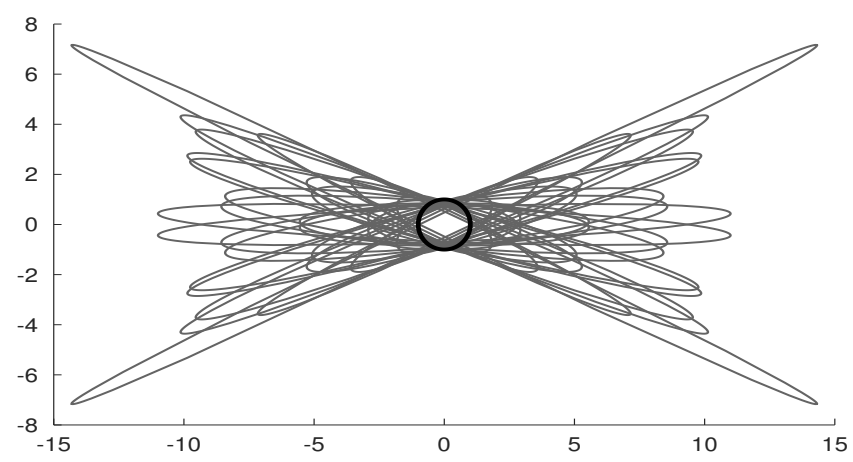

Fig. 2. Sets $\Omega_{0}$ and preimages for two-modal system with (25) after 4 iterations.

Example 2 Consider the system (1) with two modes related to the nonsingular matrices

$$
A_{1}=\left[\begin{array}{cc}
0.64 & -0.28 \\
-0.28 & 1.06
\end{array}\right], \quad A_{2}=\left[\begin{array}{ll}
0.64 & 0.28 \\
0.28 & 1.06
\end{array}\right]
$$


Both matrices have eigenvalues 0.5 and 1.2 ; the eigenvector of 0.5 for $A_{1}$ is $(2,1)$ whereas for $A_{2}$ is $(2,-1)$; the eigenvectors of 1.2 are $(-1,2)$ and $(-1,-2)$ for $A_{1}$ and $A_{2}$, respectively. If we apply the Algorithm 1 with $\Omega_{0}=\mathscr{B}^{2}$ and no constraint on the switches (or, equivalently, with automaton that generates a language formed by every possible sequence) we obtain the set in Figure 2. It can be noticed that the set $\Omega_{0}$ is not contained in the union of preimages, neither for higher horizons. Moreover, it could be inferred

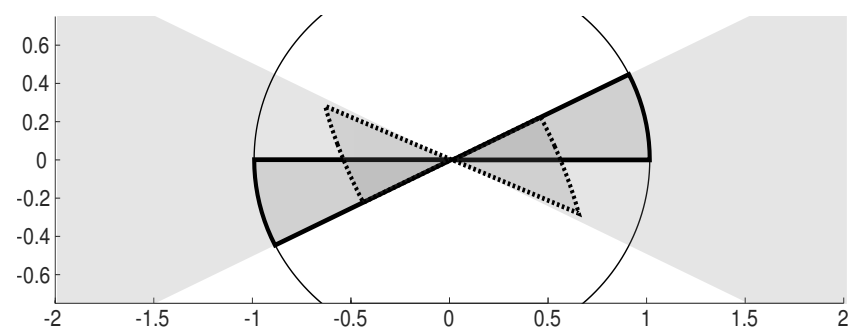

Fig. 3. Sets $S$ in light gray; $S_{+} \cap \mathscr{B}^{2}$, in bold; and $A_{1}\left(S_{+} \cap \mathscr{B}^{2}\right)$ in dotted line.

from Figure 2 that initial states in the sectors contained between the stable eigenvectors, i.e.

$$
S=\left\{x \in \mathbb{R}^{2}: x^{T} M_{S} x \geq 0\right\}, \quad \text { with } \quad M_{S}=\left[\begin{array}{cc}
1 & 0 \\
0 & -4
\end{array}\right]
$$

could be made converge to the origin. This can be formally proved by analyzing the Figure 3. Indeed, the points in $S_{+} \cap$ $\mathscr{B}^{2}$, where $S_{+}=\left\{x \in \mathbb{R}^{2}: x^{T} M_{S_{+}} x \geq 0\right\}$, with $M_{S_{+}}=\left[\begin{array}{cc}0 & 1 \\ 1 & -4\end{array}\right]$, drawn in bold line in Figure 3, are mapped in the dotted set if the mode 1 is applied. This means that for all $x \in$ $S_{+} \cap \mathscr{B}^{2}$ the successor $A_{1} x$ is in $S$ and $\left\|A_{1} x\right\| \leq \lambda\|x\|$ with $\lambda=0.7$. Analogous considerations hold for $S_{-}=\left\{x \in \mathbb{R}^{2}\right.$ : $\left.x^{T} M_{S_{-}} x \geq 0\right\}$, with $M_{S_{-}}=\left[\begin{array}{cc}0 & -1 \\ -1 & -4\end{array}\right]$, under the mode 2, i.e. for all $x \in S_{-} \cap \mathscr{B}^{2}$ one has $A_{2} x \in S$ and $\left\|A_{2} x\right\|<\lambda\|x\|$. Note that $S_{-}=\operatorname{cl}\left(S \backslash S_{+}\right)$. Hence, the successor $x_{1}^{v}$ of every $x \in S$ is still in $S$ and such that $\left\|x_{1}^{v}\right\| \leq \lambda\|x\|$ under the switching law

$$
v(x) \in \begin{cases}\{1\}, & \text { if } x \in S_{+}, \\ \{2\}, & \text { if } x \in S_{-},\end{cases}
$$

defined in $S$. Thus every trajectory starting in $S$ under control law (26) remains in $S$ and converges exponentially to the origin. This is also proved by the following inequalities: $A_{1}^{T} M_{S} A_{1}-1.2 M_{S_{+}} \geq 0$ and $A_{2}^{T} M_{S} A_{2}-1.2 M_{S_{-}} \geq 0$ that means $S$ is invariant under control (26); and $\lambda^{2} I-A_{1}^{T} A_{1}-$ $0.431 M_{S_{+}} \geq 0$ and $\lambda^{2} I-A_{2}^{T} A_{2}-0.431 M_{S_{-}} \geq 0$ that implies the decreasing of the Euclidean norm for $x \in S$ under (26).

On the other hand the initial states contained between the unstable eigenvectors, that is in

$$
I=\left\{x \in \mathbb{R}^{2}: x^{T} M_{I} x \geq 0\right\}, \quad \text { with } \quad M_{I}=\left[\begin{array}{cc}
-4 & 0 \\
0 & 1
\end{array}\right],
$$

cannot be made converge to the origin, but they diverge for any mode. Indeed the sector I, is invariant no matter on the mode applied. In fact the inequalities $A_{1}^{T} M_{I} A_{1}-0.6 M_{I} \geq 0$ and $A_{2}^{T} M_{I} A_{2}-0.6 M_{I} \geq 0$ are equivalent to invariance of $I$ for both modes 1 and 2. Moreover there exists a function that is quadratic and definite positive in I that increases along the all the possible trajectories starting in I. In fact, given $P_{I}=\left[\begin{array}{rr}-1 & 0 \\ 0 & 1\end{array}\right]$, the inequality $P_{I}-0.3 M_{I}>0$ implies positive definiteness of $V_{I}(x)=x^{T} P_{I} x$ in $I$, and the inequalities $A_{1}^{T} P_{I} A_{1}-P_{I}>0$ and $A_{2}^{T} P_{I} A_{2}-P_{I}>0$ mean that $V_{I}(x)$ increases along every possible trajectory starting in $I$.

Therefore, summarizing, all the trajectories starting in $S$ exponentially converge to the origin under the switching law (26), but the switched system is not globally exponentially stabilizable. On the other hand, appropriate initial switching sequences can be designed such that every initial state can be steered in finite time in the sector $S$. Consider for instance the additional modes $A_{3}=\lambda R(\pi / 4), A_{4}=\lambda R(\pi / 2)$ and $A_{5}=\lambda R(3 \pi / 4)$ and the automaton in Figure 4. Therefore,

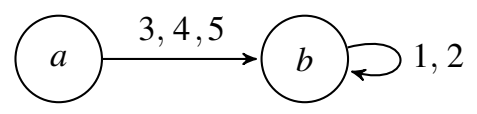

Fig. 4. Automaton of Example 2.

if the initial state is not in $S$, an appropriate rotation can be performed through $A_{3}, A_{4}$ or $A_{5}$ to steer the state in $S$. Then the control switching law can be applied to generate an exponentially convergent trajectory, with decay rate $\lambda$. Thus, the system is GES but it does not admit any ECLF as in Definition 3.

The counterexample above implies that recurrent ECLF are only sufficient for GES. The relations between the different stabilizability properties are summarized in Figure 5.

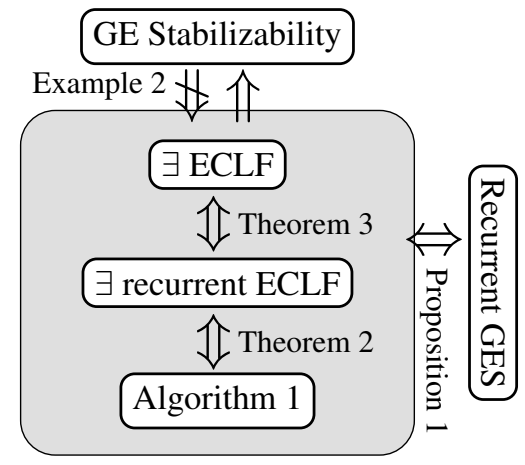

Fig. 5. Stabilizability relations diagram

\section{Dependence on automaton initial state}

In the previous sections we implicitly considered that the stabilizability of switched systems subject to language constraints does not depend on the set $\mathscr{S}_{0}$ of automaton initial conditions, see Remark 1. Hereafter, the dependence on $\mathscr{S}_{0}$ of the existence of an ECLF and of the stabilizability are analyzed. 


\subsection{Recurrent ECLF}

First we prove that the existence of an ECLF does not depend on $\mathscr{S}_{0}$, provided Assumption 2 is satisfied.

Proposition 4 Let Assumption 1 hold. An exponential control Lyapunov function for the system (1) under language constraint $L(\mathscr{A})$ exists with $\mathscr{S}_{0}=\mathscr{S}$ if and only if it exists with $\mathscr{S}_{0} \subseteq \mathscr{S}$ satisfying Assumption 2.

Proof: About sufficiency, note that an ECLF for a particular set of initial automaton states $\mathscr{S}_{0} \subseteq \mathscr{S}$, is an ECLF also for initial state set $\mathscr{S}$. To prove necessity, suppose that an ECLF in a non-empty $\mathscr{R} \subseteq \mathscr{S}$ exists. From Theorems 2 and 3 we can suppose that the Lyapunov function $V(x, r)$ is defined as (15) in $\mathscr{R}$. For every $\mathscr{S}_{0} \subseteq \mathscr{S}$ such that Assumption 2 holds, we prove that there exists also an ECLF in $\mathscr{R}_{0} \subseteq \mathscr{S}$ such that $\mathscr{R}_{0} \cap \mathscr{S}_{0} \neq \emptyset$. Consider a node $s_{0} \in \mathscr{S}_{0}$ such that $\mathscr{R}$ is reachable from $s_{0}$, that exists from Assumption 2. Denote with $s_{r}$ one node in $\mathscr{R}$ reachable through the shortest path from $s_{0}$ and $\mathscr{R}$ and $\sigma^{0}$ the switching sequence generated by such path. Thus, the path from $s_{0}$ and $s_{r}$ does not contain any node in $\mathscr{R}$ except $s_{r}$. Denote $p=\left|\sigma^{0}\right|$ and define $m \in \mathscr{S}^{p+1}$ such that $m_{1}=s_{0}, m_{p+1}=s_{r}$ and $m_{k+1} \in \delta\left(m_{k}, \sigma_{k}^{0}\right)$ for all $k \in \mathbb{N}_{p}$, i.e. $m$ is the automaton trajectory starting in $s_{0}$ terminating in $s_{r}$ and generating $\sigma^{0}$. Since the path is the shortest, then $m_{j} \neq m_{k}$ for all $j, k \in \mathbb{N}_{p}$ such that $j \neq k$. Given the set $\Omega^{s_{r}, s}$ that determines $V\left(x, s_{r}\right)$, define the sets

$$
\Omega^{m_{k}, s}= \begin{cases}\mu A_{\sigma_{k}^{0}}^{-1} \Omega^{s_{r}, s}, & \text { if } \quad k=p \\ \mu A_{\sigma_{k}^{0}}^{-1} \Omega^{m_{k+1}, s}, & \text { if } k \in \mathbb{N}_{p-1}\end{cases}
$$

and the control policy $v\left(x, m_{k}\right)=\left(\sigma_{k}^{0}, m_{k+1}\right)$ on $\mathbb{R}^{n} \times m_{k}$ for all $k \in \mathbb{N}_{p}$. The sets (27) are $\mathrm{C}^{*}$-sets from Assumption 1 and since $\Omega^{s_{r}, s}$ is a $\mathrm{C}^{*}$-set. Then, the function $V(x, r)$ defined as in (15) on $\mathscr{R}_{0}=\mathscr{R} \cup \bigcup_{k \in \mathbb{N}_{p}} m_{k}$ by the sets (9) and (27), satisfies $(i)$ in Definition 3 since $\Omega^{m_{k}, s}$ are $C^{*}$-sets and (ii) since it decreases of $\mu$ by applying $v(x, r)$, by construction. Hence, $V(x, r)$ is an ECLF in $\mathscr{R}_{0}$, where $s_{0}=m_{1} \in \mathscr{R}_{0} \cap \mathscr{S}_{0}$.

Proposition 4 implies that the existence of an ECLF for the system (1) satisfying Assumptions 1 and 2 under language constraint $L(\mathscr{A})$ does not depend on the initial state of the automaton.

\subsection{Recurrent stabilizability}

In this section we prove that, although from Assumption 2 every mode is reachable from an initial automaton state, stabilizability depends on $\mathscr{S}_{0}$.

Example 3 Consider the systems of Example 2 with additional modes 6,7 and 8 and related matrices $A_{6}=A_{3}^{-1}$,
$A_{7}=A_{4}^{-1}$ and $A_{8}=A_{5}^{-1}$. The automaton determining the language constraints is depicted in Figure 6. Every node is reachable from every other node but the global stabilizability of the system depends on the initial state of the automaton. Indeed, if $\mathscr{S}_{0}=\{d\}$, therefore the system is not stabilizable since if the initial state is in the sector I, then it cannot be steered in $S$. In fact the rotations related to modes 3,4 and 5 would be preceded by the inverse rotations, i.e. 6,7 and 8 respectively, leading to a null effect. On the other hand if $\mathscr{S}_{0}=\mathscr{S}$ then the appropriate initial rotation could be performed to steer the state in $S$, if necessary, and thus the system would be stabilizable.

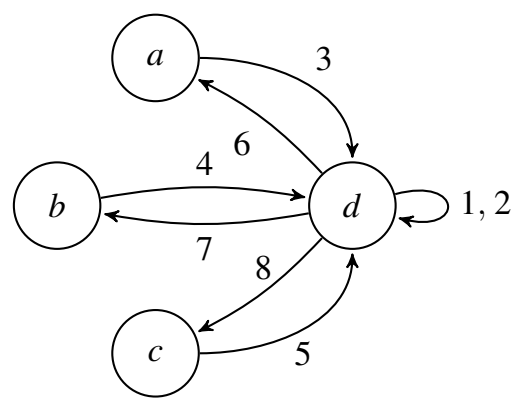

Fig. 6. Automaton of Example 3.

Example 3 implies that the stabilizability of the system (1) satisfying Assumptions 1 and 2 under language constraint $L(\mathscr{A})$ depends on the initial state of the automaton.

\section{Examples}

Example 4 Consider the 2-dimensional switched system with 2 unstable modes

$$
A_{1}=\left[\begin{array}{cc}
1.2 & 0 \\
0 & 0.5
\end{array}\right], \quad A_{2}=1.1 R(\pi / 3),
$$

and constraints determined by the 5-states automaton of Figure 7. The Algorithm 1 has been applied for every $s \in \mathscr{I}$.

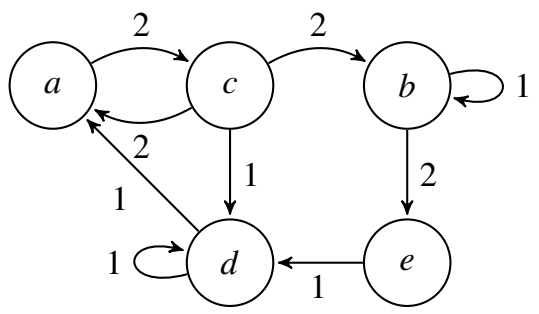

Fig. 7. Automaton of Example 4.

No recurrent ECLF has been obtained after 5 steps, but for $N^{s}=6$ the stop condition holds for three automaton states, i.e. for $a, c$ and $d$. The resulting sets are drawn in Figure 8. The control policy related to the mode $s=d$ has 
been applied, resulting in the state and automaton trajectories shown in Figure 9. Note that, since we applied a minswitching control strategy, given in the proof of Proposition 2, the recurrence in $d$ is not assured every 6 steps or less, see the instants between 17 and 24.
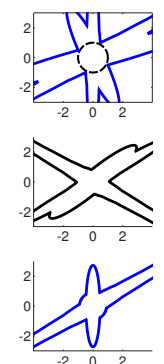

$\int^{2} L$
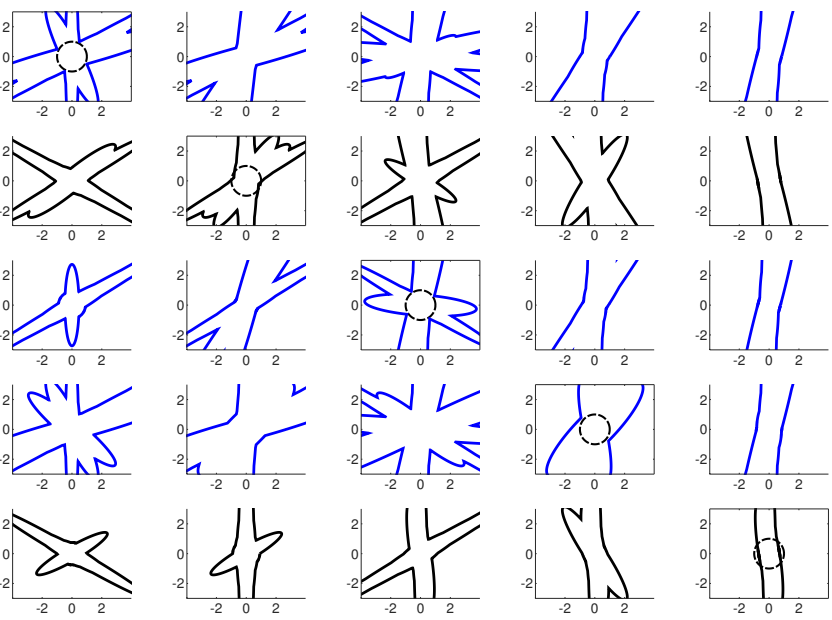

Fig. 8. Sets $\Omega^{r, s}$ for all $r, s \in \mathscr{I}$ and $N^{s}=6$. The set $\Omega^{r, s}$ is depicted in the line $s$ column $r$. In blue the sets related to recurrent ECLF.
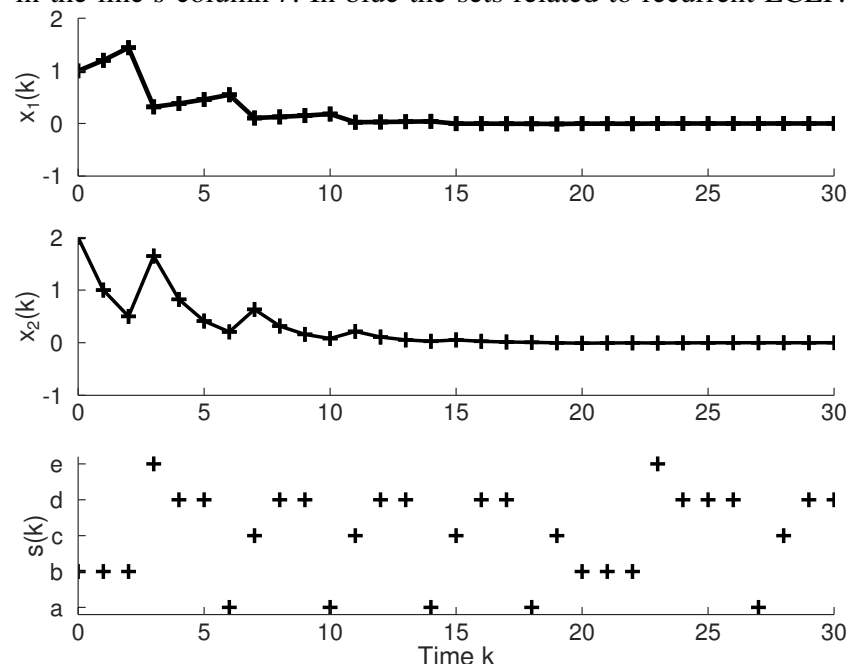

Fig. 9. Evolutions of the systems states, top and middle, and automaton state, bottom.

Example 5 Consider Example 7 in [9], that is (1) with

$$
A_{1}=\left[\begin{array}{ccc}
1.2 & 0 & 0 \\
-1 & 0.8 & 0 \\
0 & 0 & 0.5
\end{array}\right], \quad A_{2}=\left[\begin{array}{ccc}
0.7 & 0 & 0 \\
0 & -0.6 & -2 \\
0 & 0 & -1.2
\end{array}\right]
$$

to whose switching we impose the constraints given by the automaton in Figure 10. This means that, in practice, the sequential repetition of mode 2 is forbidden. The Algorithm 1 applied with $s=b$ and $\Omega_{0}=\mathscr{B}^{2}$, generates the sequence of $\Omega_{j}^{s}$ depicted in Figure 11 and then stops after 4 iteration, while the analogous algorithm without any constraint terminates in 3 steps, see [9].

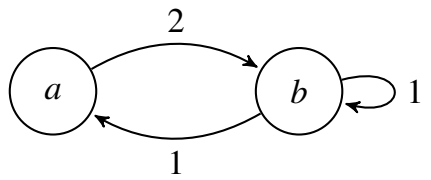

Fig. 10. Automaton of Example 5.

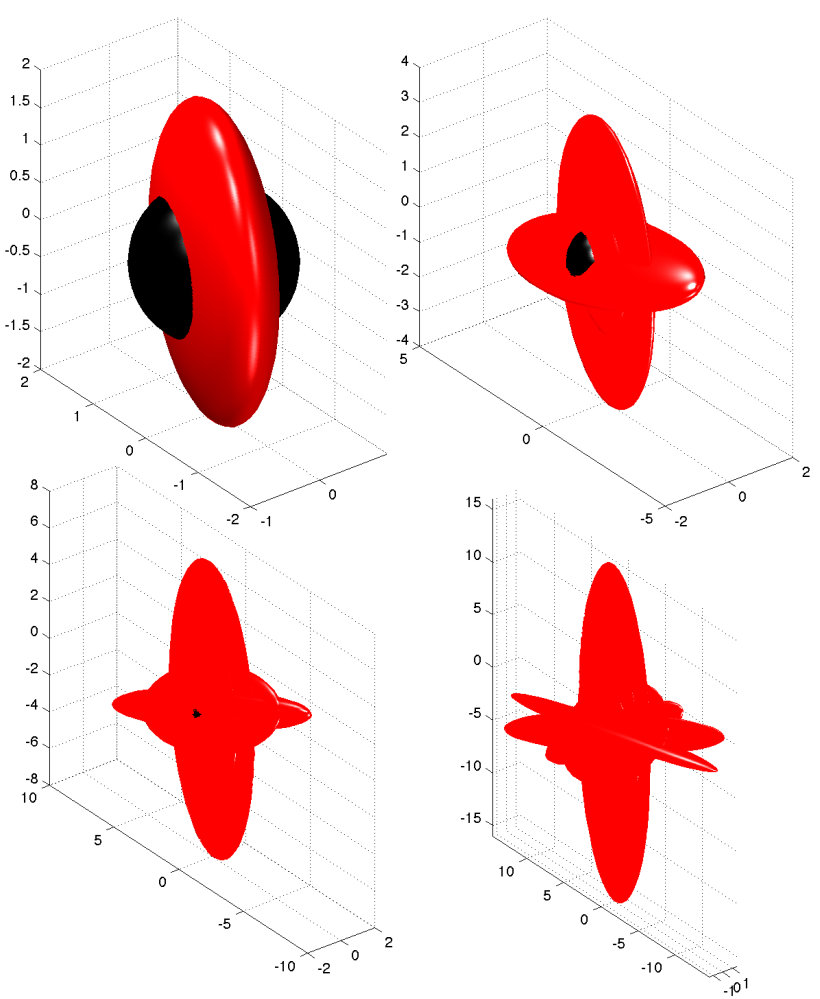

Fig. 11. Sets $\mathscr{B}^{2}$ in black and $\Omega_{j}^{s}$ for $j \in \mathbb{N}_{4}$, in red.

\section{Conclusions}

In this paper we presented a constructive approach to characterize stabilizability and recurrent stabilizability for switched linear systems subject to constraints on the switching law. An algorithm is proposed whose finite termination provides a necessary and sufficient condition for the existence of an ECLF. Moreover, the exact relations between stabilizability, recurrent stabilizability and existence of ECLF are provided. The dependence of stabilizability on the automaton initial state is also proved.

\section{Acknowledgement}

This work was partially supported by project ANR COMPACS - "Computation Aware Control Systems", ANR-13BS03-004. 


\section{References}

[1] A. A. Ahmadi, R. M. Jungers, P. A. Parrilo, and M. Roozbehani. Joint spectral radius and path-complete graph Lyapunov functions. SIAM Journal on Control and Optimization, 52(1):687-717, 2014.

[2] N. Athanasopoulos, K. Smpoukis, and R. M. Jungers. Invariance in constrained switching. arXiv preprint arXiv:1702.00598, 2017.

[3] C. Baier, J.-P. Katoen, et al. Principles of model checking, volume 26202649. MIT press Cambridge, 2008.

[4] F. Blanchini. Nonquadratic Lyapunov functions for robust control. Automatica, 31:451-461, 1995.

[5] F. Blanchini and S. Miani. Set-Theoretic Methods in Control. Birkhäuser, 2008.

[6] F. Blanchini and C. Savorgnan. Stabilizability of switched linear systems does not imply the existence of convex Lyapunov functions. Automatica, 44:1166 - 1170, 2008.

[7] J. Daafouz, P. Riedinger, and C. Iung. Stability analysis and control synthesis for switched systems: A switched Lyapunov function approach. IEEE Transactions on Automatic Control, 47:1883-1887, 2002.

[8] M. Fiacchini, A. Girard, and M. Jungers. On the stabilizability of discrete-time switched linear systems: novel conditions and comparisons. IEEE Transactions on Automatic Control, 61(5):11811193, 2016.

[9] M. Fiacchini and M. Jungers. Necessary and sufficient condition for stabilizability of discrete-time linear switched systems: A set-theory approach. Automatica, 50(1):75 - 83, 2014.

[10] M. Fiacchini, M. Jungers, and A. Girard. Exponential stabilization of language constrained discrete-time switched linear systems: A geometrical approach. In 2016 European Control Conference (ECC), pages 2035-2040, 2016.

[11] J. C. Geromel and P. Colaneri. Stability and stabilization of discretetime switched systems. International Journal of Control, 79(7):719728, July 2006.

[12] W. P. M. H. Heemels, A. Kundu, and J. Daafouz. On LyapunovMetzler inequalities and S-procedure characterisations for the stabilisation of switched linear systems. IEEE Transactions on Automatic Control, PP(99):1-1, 2016.

[13] M. Jungers, A. Girard, and M. Fiacchini. Language constrained stabilization of discrete-time switched linear systems: a LyapunovMetzler inequalities approach. In 2016 IEEE 55th Conference on Decision and Control (CDC), pages 5539-5544. IEEE, 2016.

[14] J.-W. Lee and G. E. Dullerud. Uniformly stabilizing sets of switching sequences for switched linear systems. IEEE Transactions on Automatic Control, 52(5):868-874, 2007.

[15] D. Liberzon. Switching in Systems and Control. Birkhäuser, Boston, MA, 2003.

[16] H. Lin and P. J. Antsaklis. Stability and stabilizability of switched linear systems: a survey of recent results. IEEE Transaction on Automatic Control, 54(2):308-322, 2009.

[17] A. P. Molchanov and Y. S. Pyatnitskiy. Criteria of asymptotic stability of differential and difference inclusions encounterd in control theory. Systems \& Control Letters, 13:59-64, 1989.

[18] M. Philippe and R. Jungers. Converse Lyapunov theorems for discrete-time linear switching systems with regular switching sequences. In 2015 European Control Conference (ECC), pages 1902-1907, 2015.

[19] A. M. Rubinov and A. A. Yagubov. The space of star-shaped sets and its applications in nonsmooth optimization. In V.F. Demyanov and L.C.W. Dixon, editors, Quasidifferential Calculus, pages 176202. Springer Berlin Heidelberg, 1986.
[20] E. De Santis and M. D. Di Benedetto. Theory and computation of discrete state space decompositions for hybrid systems. European Journal of Control, 19(1):1-10, 2013.

[21] M. Sharir. A strong connectivity algorithm and its applications to data flow analysis. Computers and Mathematics with Applications, 7(1):67-72, 1981.

[22] Z. Sun and S. S. Ge. Stability Theory of Switched Dynamical Systems. Springer, 2011.

[23] R. E. Tarjan. Depth-first search and linear graph algorithms. SIAM Journal on Computing, 1(2):146-160, 1972.

[24] Y. Wang, N. Roohi, G. E. Dullerud, and M. Viswanathan. Stability of linear autonomous systems under regular switching sequences. In IEEE 53rd Annual Conference on Decision and Control (CDC), pages 5445-5450. IEEE, 2014.

[25] G. Weiss and R. Alur. Automata based interfaces for control and scheduling. In Hybrid Systems: Computation and Control, pages 601-613. Springer, 2007.

[26] W. Zhang, A. Abate, J. Hu, and M. P. Vitus. Exponential stabilization of discrete-time switched linear systems. Automatica, 45(11):25262536, 2009.

\section{A Digraphs and Strongly Connected Components}

Definition 9 Let $(s, r) \in \mathscr{V}^{2}$. The nodes $s$ and $r$ are strongly connected if $s=r$ or if there exist a directed path from $s$ to $r$ and a directed path from $r$ to $s$. This relation between nodes is reflexive, symmetric and transitive and it is then an equivalence relation on the nodes.

This equivalence relation allows to partition $\mathscr{V}$ of $\mathscr{G}$ into disjoint sets called Strongly Connected Components defined in Definition 10.

Definition 10 [Strongly Connected Components (SCCs).] Let $\mathscr{G}=(\mathscr{V}, \mathscr{E})$ be a finite digraph and $\mathscr{C} \subseteq \mathscr{V} . \mathscr{C}$ is strongly connected if for every pair of nodes $(s, r) \in \mathscr{C}^{2}, s$ and $r$ are strongly connected. A strongly connected component (SCC) of the digraph $\mathscr{G}$ is a maximally strongly connected set of nodes. In other words, $\mathscr{C}$ is a SCC if $\mathscr{C}$ is strongly connected and if there does not exist a SCC distinct from $\mathscr{C}$ which includes $\mathscr{C}$. A SCC $\mathscr{C}$ is called trivial if $\mathscr{C}=\{s\}$ and $(s, s) \notin \mathscr{E}$. A SCC $\mathscr{C}$ is called terminal if there is no SCC $\mathscr{D} \neq \mathscr{C}$ such that $(s, r) \in \mathscr{E}$ for some $s \in \mathscr{C}$ and $r \in \mathscr{D}$. The finite digraph $\mathscr{G}$ is cyclic if and only if $\mathscr{G}$ contains a nontrivial SCC.

Every node of the digraph $\mathscr{G}$ belongs to one and only one SCC: the SCCs are equal or disjoint. In the following, we will denote $\mathscr{C}_{i}$ with $i \in \mathbb{N}_{d}$, the $d$ SCCs of the digraph $\mathscr{G}$ and $p_{i}=\left|\mathscr{C}_{i}\right|$. We define the condensation of $\mathscr{G}$ as follows.

Definition 11 The condensation of a digraph $\mathscr{G}$ is a digraph $\mathscr{G}_{S C C}=\left(\mathscr{V}_{S C C}, \mathscr{E}_{S C C}\right)$ built as follows. Each $S C C \mathscr{C}_{i}$ is condensed into a single node $v_{i} \in \mathscr{V}_{S C C}$ and then $\left|\mathscr{V}_{S C C}\right|=d$. The set of condensed edges is $\mathscr{E}_{S C C}=\left\{\left(v_{i}, v_{j}\right) \in \mathscr{V}_{S C C}^{2}: \exists(r, s) \in\right.$ $\left.\mathscr{E}, r \in \mathscr{C}_{i}, s \in \mathscr{C}_{j}, i \neq j\right\}$

In practice $\mathscr{E}_{S C C}$ consists in the directed edges of $\mathscr{G}$ making the link between the SCCs. The condensation $\mathscr{G}_{S C C}$ is a 
Directed Acyclic Graph (DAG), because it does not contain any cycle. We can define a relation between the SCCs as $\mathscr{C}_{i} \succeq \mathscr{C}_{j}$ if there exists a path between one node in $\mathscr{C}_{i}$ and a node in $\mathscr{C}_{j}$. This is a partial relation order between the SCCs because it is reflexive $\left(\mathscr{C}_{i} \succeq \mathscr{C}_{i}\right)$, antisymmetric $\left(\mathscr{C}_{i} \succeq \mathscr{C}_{j}\right.$ and $\mathscr{C}_{j} \succeq \mathscr{C}_{i}$ imply $\left.\mathscr{C}_{i}=\mathscr{C}_{j}\right)$ and transitive $\left(\mathscr{C}_{i} \succeq \mathscr{C}_{j}\right.$ and $\mathscr{C}_{j} \succeq \mathscr{C}_{\ell}$ imply $\mathscr{C}_{i} \succeq \mathscr{C}_{\ell}$ ).

Computing the condensation of a digraph is a standard task and several dedicated algorithms are available in the literature. Among them, one can cite the Kosaraju's algorithm [21] or the Tarjan's algorithm [23].

Proposition 5 Let $\mathscr{G}$ be the digraph associated with the automaton $\mathscr{A}$ and its condensation $\mathscr{G}_{S C C}$. Every trajectory of the constrained switched system subject to the language constraint $L(\mathscr{A})$, has a projection on the automaton state space that ultimately enters and does not exit a nontrivial SCC.

Proof: The result is due to the fact that the condensation is a directed acyclic graph. 